\title{
Die frische Achillessehnenruptur - operative Behandlungstechniken
}

\author{
Gerhard J. Bauer
}

\section{Zusammenfassung}

In den letzten Jahren wird eine deutliche Zunahme der Achillessehnenrupturen beobachtet. Trotz zahlreicher Behandlungsmöglichkeiten gibt es noch keine allgemeingültige Managementstrategie. Die konservativ-funktionelle Therapie hat sich gegenüber der immobilisierenden Behandlung durchgesetzt. Hauptprobleme der konservativen Behandlung bleiben eine höhere Rerupturrate und die Ausheilung der Sehne in Verlängerung bei nicht absolut konsequenter Durchführung der Behandlung. Die Vorteile der operativen Therapie sind eine bessere Primärstabilität und damit etwas bessere frühfunktionelle Ergebnisse. Als Nachteil eines operativen Eingriffs müssen immer mögliche Komplikationen wie Wundheilungsstörungen bedacht werden, die bis zum Verlust der Sehne führen können. Man unterscheidet offene, kombiniert offen-perkutane und perkutaneTechniken. Insbesondere die perkutanen Techniken haben sich weiterentwickelt und sind bei guter Adaption der Sehnenenden unter Erhalt des Epitendineums für Patienten mit Belastungsanspruch in Beruf und Sport empfehlenswert. Dennoch ist die Behandlung individuell an den Bedürfnissen und Risiken des Patienten auszurichten.

\section{Acute Achilles Tendon Rupture - Operative Techniques}

A significant increase in Achilles tendon ruptures has been observed in the past few years. Although numerous treatment options are available, there is still no generally accepted management strategy. Conservative functional treatment has prevailed over immobilisation treatment. The main problems associated with conservative treatment are a higher rate of repeated ruptures and tendon healing in elongation if the treatment is not carried out consistently. The advantage of operative treatment is improved primary stability, which may lead to slightly improved functional results in the first months of recovery. The main disadvantage of operative procedures is the possibility of complications such as impaired wound healing, which may even lead to the loss of the tendon. There are open, open percutaneous, and percutaneous techniques. Percutaneous techniques in particular have been refined and are recommended for patients with a physically demanding job or patients engaging in strenuous physical exercise, provided that the tendon ends are precisely apposed and the epitendineum has been preserved. Nonetheless, treatment should be individually adapted to the needs and possible risk factors of the patient.

\section{Einleitung}

Studien aus Skandinavien und den USA berichten über eine deutliche Zunahme der Achillessehnenrupturen in den letzten 10 Jahren. Die überwiegende Mehr-

OP-JOURNAL 2015; 31: 188-192

(c) Georg Thieme Verlag KG Stuttgart · New York DOI http://dx.doi.org/10.1055/s-0041-110061

Die Kombination einer unvorhergesehenen Anspannung mit degenerativen Veränderungen in der Sehne - z.B. ein Tritt in ein Loch - gilt als häufigste Ursache einer Ruptur. Direkte Traumen dagegen, wie Tritte bei Kontaktsportarten, sind selten $[17,21]$.

Für die Routinediagnostik der Achillessehnenruptur sind Anamnese, Klinik, dynamische Sonografie und Röntgenbild völlig ausreichend. Ein MRT ist nur in Ausnahmefällen oder bei veralteten Achillessehnenrupturen erforderlich [11, 17].

Trotz zahlreicher in der Literatur beschriebener Behandlungsmöglichkeiten - über 100 Publikationen seit 2014 - gibt es noch keine allgemeingültige Managementstrategie. Die Meinungen sind insbesondere geteilt, wenn es um die Frage operatives oder konservatives Vorgehen geht $[9,11,14]$.

Keine allgemein gültige Therapiestrategie.

Um eine Therapieempfehlung aussprechen zu können, müssen neben den individuellen Patientengegebenheiten die Besonderheiten der verschiedenen Behandlungen mit ihren Risiken berücksichtigt werden.

Bei der konservativ-immobilisierenden Therapie besteht ein hohes Risiko einer Reruptur (bis 30\%) oder die Gefahr einer in Verlängerung ausheilenden Sehne (Abb. 1), die konservativ-funktionelle Therapie erfordert eine hohe Compliance des Patienten $[1,20]$. Grundsätzlich muss die konservative Therapie immer als Option angeboten werden. Die am meisten gefürchtete Komplikation nach Operation ist der postoperative Infekt, der bis zum kompletten Verlust der Sehne mit anschließend aufwendiger plastischer Deckung führen kann $[2,4,12]$.
Die meisten Achillessehnenrupturen Tendenz steigend - erleiden ältere männliche Sportler. sich bei sportlicher Betätigung von Männern zwischen 30 und 50 Jahren, wenn auch für die Zunahme vor allem die älteren Sporttreibenden verantwortlich sind $[13,17,18]$. 

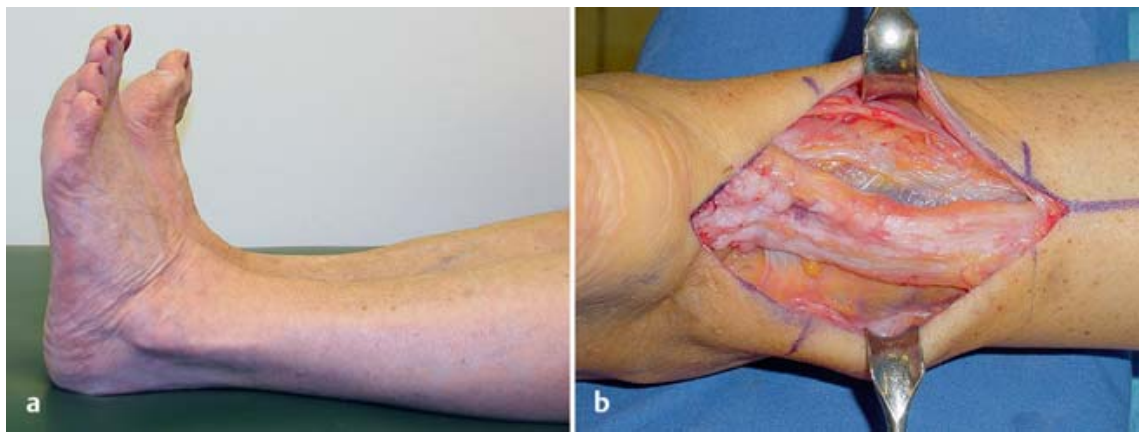

Abb. 1 a und $\mathbf{b}$ a Vermehrte Dorsalextension des rechten Fußes durch die in Verlängerung ausgeheilte konservativ behandelte Ruptur. b Das intraoperative Bild zeigt die Achillessehne als funktionslosen Strang ohne Vorspannung.

In Deutschland war bis Mitte der 90erJahre die offene Sehnennaht die am häufigsten eingesetzte Behandlung [15]. Die Entwicklung minimalinvasiver Operationstechniken hat besonders in der letzten Dekade die therapeutischen Möglichkeiten deutlich erweitert, und sie finden immer mehr Einzug in den operativen Alltag [1].

Im folgenden Beitrag werden die aktuellen Techniken der operativen Behandlung akuter Achillessehnenrupturen dargestellt.

\section{Operative Techniken}

\section{Allgemein}

Ziel aller operativen Techniken ist die Adaption der rupturierten Sehnenenden in der ursprünglichen Spannung und deren sichere Naht bis zur Ausheilung [21]. Hat man sich für die operative Versorgung einer Ruptur entschieden, gilt es, folgende Parameter zu beachten.

Wichtige Parameter bei der operativen Versorgung sind: Alter der Ruptur, Rupturlokalisation, Retraktion der Sehnenenden.

Vor der Operation erfolgt das Messen der Plantarflexionsstellung des verletzten und des gesunden Fußes.

\section{Frische Ruptur/alte Ruptur}

Optimalerweise erfolgt die Operation in der 1 . Woche nach Verletzung. Rupturen, die älter als 4 Wochen sind, werden als alt oder chronisch bezeichnet [1]. In diesen Fällen finden wir retrahierte, degenerativ veränderte Sehnenenden, die sich meist nicht mehr spannungsfrei End-zu-End nähen lassen und somit plastische Rekonstruktionen (z.B. Griffelschachtelplastik) erforderlich machen.

\section{Rupturlokalisation}

In über $80 \%$ ist das mittlere Sehnendrittel betroffen. Während die proximalen Rupturen am Muskel-Sehnen-Übergang eine Domäne der konservativ-funktionellen Therapie sind, erfordern die seltenen Rupturen im distalen Drittel einen operativen Eingriff [1] (Abb. 2).

\section{Retraktion der Sehnenenden}

Mit der dynamischen Sonografie kann vor der Operation geprüft werden, ob sich die Sehnenstümpfe annähern oder ob durch das häufig vorhandene Hämatom ein größerer $(>10 \mathrm{~mm})$ Spalt verbleibt, der gegen ein minimalinvasives Vorgehen spricht $[1,11]$.

Ziel aller operativen Techniken ist die Adaption der rupturierten Sehnenenden in der ursprünglichen Spannung und deren sichere Naht bis zur Ausheilung [21].

Alle Eingriffe werden, unabhängig von der gewählten Technik, in Bauchlage durchgeführt. Vor dem sterilen Abdecken empfiehlt es sich, die Plantarflexionsstellung des gesunden und des verletzten Fußes zu messen. Eine perioperative Antibiotikaprophylaxe (z.B. mit einem Cephalosporin der 2. Generation) ist empfehlenswert. Die Anlage einer Blutsperre erleichtert die Operation, ist jedoch nicht unbedingt erforderlich [1, $3]$.

\section{Offene Sehnennaht}

Zur Schonung des lateral verlaufenden $\mathrm{N}$. suralis empfiehlt sich der dorsomediale Zugang. Inzisionen, die die Achillessehne kreuzen, sollten vermieden werden, da sie bei evtl. später erforderlichen Revisionen das Risiko von Komplikationen erhöhen.

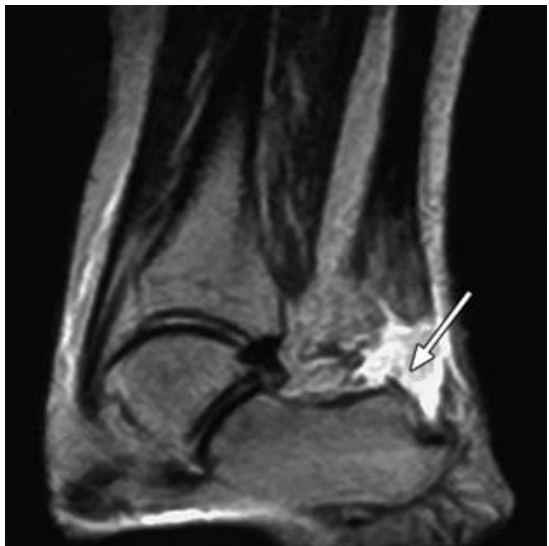

Abb. 2 Distale Ruptur (Pfeil) mit Retraktion der Sehne im MRT.

Grundsätzlich unterscheidet man bei den offenen Nähten adaptierende und mehr belastbare Techniken $[1,12]$. In der Literatur sind hierzu zahlreiche Verfahren beschrieben. Bewährt haben sich End-zu-End-Nähte in den Techniken nach Bunnell, Kirchmayr-Kessler oder Krackow [17]. Bei der Wahl des Nahtmaterials sollte man bedenken, dass sehr kräftige Fäden zwar die primäre Reißfestigkeit, aber auch das Risiko von Heilungsstörungen erhöhen [4]. Zur Anwendung kommen, ohne nachweisbare Unterschiede, langsam resorbierbare (z.B. PDS II der Stärke USP 1, Fa. Ethikon, Norderstedt) oder nicht resorbierbare Fäden (z. B. FiberWire, Fa. Arthrex, Naples) [17]. In geeigneten Fällen kann eine kräftige Rahmennaht mit einer Feinadaption der Sehnenenden kombiniert werden, oder man nimmt eine vorhandene Plantarissehne zur Augmentation. Wenn möglich, sollte man versuchen, das Epitendineum $\mathrm{zu}$ verschließen, elementar wichtig ist jedoch die spannungsarme Hautnaht (Abb. 3).

Eine spannungsfreie Hautnaht ist die beste Infektionsprophylaxe.

Eine Sonderstellung nehmen die distalen ansatznahen Rupturen bzw. Abrisse der Achillessehne ein. Im eigenen Patientengut beträgt die Häufigkeit 8,5\%; das Durchschnittsalter dieser Patienten war mit 50 Jahren fast 10 Jahre höher als das des Gesamtkollektivs. In diesen Fällen hat sich die Refixation mit Nahtankern bewährt [17]. Diese Technik - aber auch die transossäre Fadenführung - ermöglicht ein kombiniert offen-perkutanes Vorgehen, indem der proximale Sehnenstumpf perkutan angeschlungen wird. Die Literatur hierzu ist äußerst spärlich. 


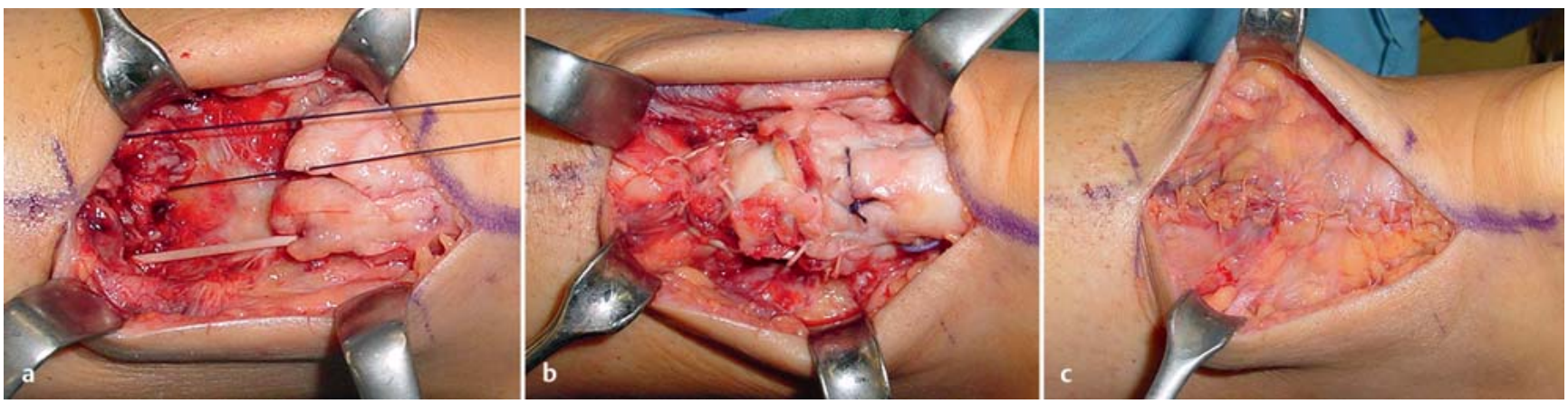

Abb. 3 a bis c a Bauchlage, rechtes Bein. Dorsomedialer Zugang zur Schonung des N. suralis. Plantarissehne vorhanden. Proximales Sehnenende retrahiert und zur Mobilisierung mit Haltefaden angeschlungen. b End-zu-End-Naht durch Kombination einer Rahmennaht (0,7-mm-PDS-Kordel, Fa. Ethikon, Norderstedt) und Feinadaption mit resobierbaren Fäden (PDS II $3 \times 0$, Fa. Ethikon, Norderstedt). c kompletter Verschluss des Epitendineums.

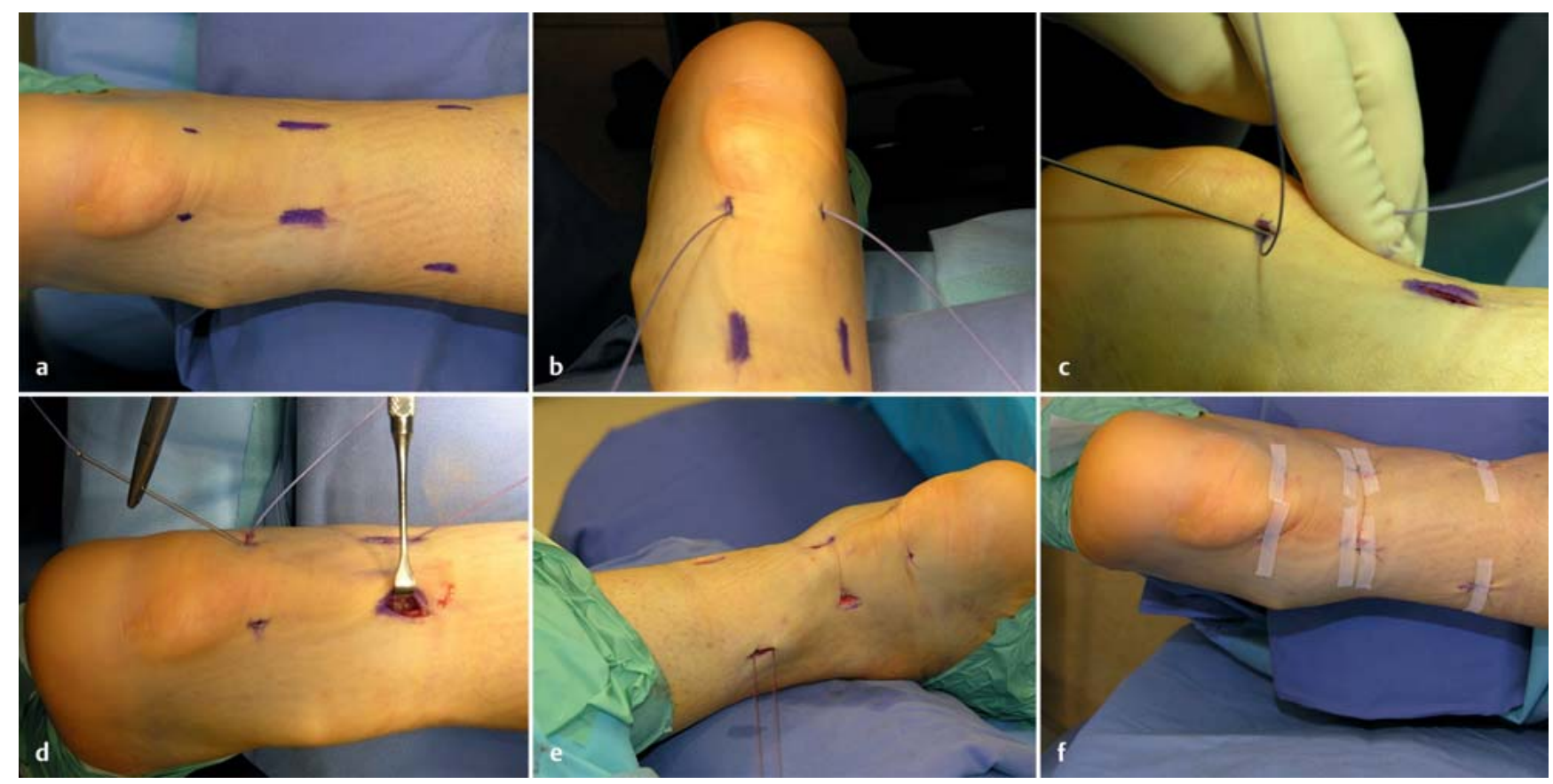

Abb. 4a bis f Modifizierte perkutane Achillessehnennaht. a Anzeichnen der 6 Hautinzisionen. b Distal quere transtendinöse Platzierung der 0,7mm-PDS-Kordel mit 2 armierten geraden Nadeln (Fa. Ethikon, Norderstedt). c Mit den beiden Nadeln von distal nach proximal Anschlingen des distalen Stumpfes. d Vor dem Anschlingen des proximalen Sehnenstumpfs Darstellen des N. suralis an der proximalen lateralen Inzision (Wundhaken). e Knüpfen der Kordel proximal medial in leichter Überkorrektur der Plantarflexion. f Hautverschluss mit atraumatischen Nähten.

\section{Minimalinvasive Sehnennaht}

Sie wurde erstmals 1977 beschrieben mit dem Ziel eines geringeren Weichteiltraumas und eines niedrigeren Infektionsrisikos im Vergleich zur offenen Naht und einer höheren Primärstabilität im Vergleich zum konservativen Vorgehen [16]. Inzwischen wurden zahlreiche Varianten publiziert, deren vorrangiges Ziel es war, die Nachteile dieser Technik - Risiko einer Schädigung des N. suralis, ungenügende Festigkeit der Naht, ungenügende Adaption der Sehnenenden - zu minimieren $[1,17]$. Wird die Rupturregion bei der Operation eröffnet, spricht man von kombiniert offen-perkutanen Techniken (z.B. Hannoveraner Technik
[12], im Gegensatz zu den perkutanen Nahttechniken ohne Eröffnung der Rupturregion [1]. Zu Letzteren gehören, neben der Originaltechnik nach Ma und Griffith [16], zahlreiche Modifikationen einschließlich einer eigenen Technik mit Darstellung des N. suralis an der proximalen lateralen Stichinzision und die perkutane Naht mit dem Dresdener Instrumentarium (Abb. 4 und 5). Ziel aller perkutanen Nahttechniken war und ist der Erhalt des Epitendineums wegen seiner zentralen Bedeutung für die überwiegend extrinsische Heilung der Sehne $[2,3]$.

Die ideale Indikation für die perkutanen Techniken ist die frische Ruptur im mitt- leren Sehnendrittel mit guter Annäherung der Sehnenenden.

Ideal bei: Verletzung im mittleren Drittel der Sehne, Operation in der 1 . Woche, gute Adaption der Sehnenenden $(<10 \mathrm{~mm})$.

Praktische Anmerkungen: Da bei den perkutanen Nähten die Sehnenenden und damit die korrekte Adaption der Sehnenstümpfe nicht mit dem Auge und palpatorisch nur unsicher kontrolliert werden können, empfiehlt sich bei Unsicherheit die sonografische Kontrolle [2]. Für ein optimales Ergebnis ist das Erreichen der korrekten Vorspannung der Sehne sehr wichtig. Die erforderliche 

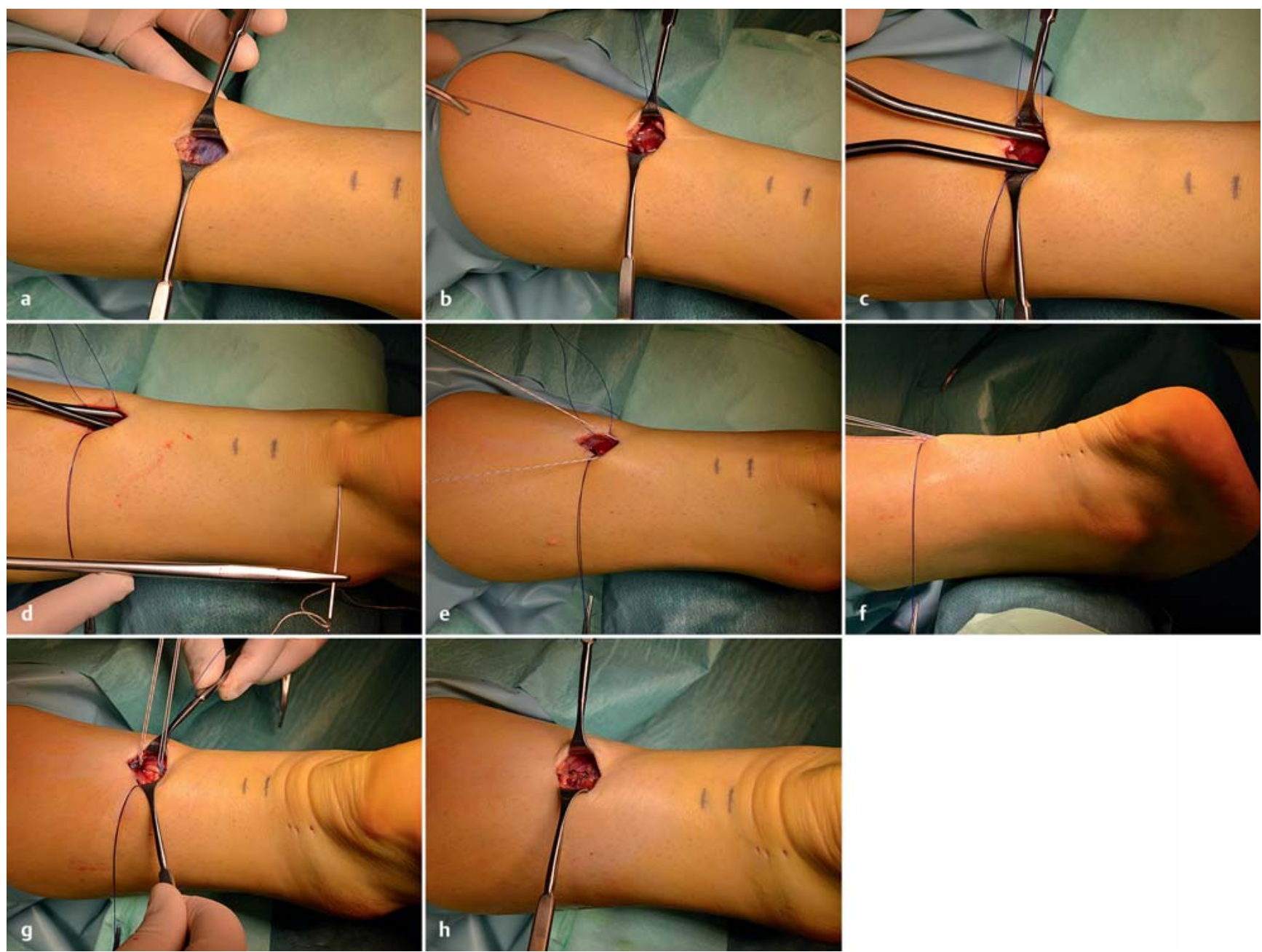

Vorspannung orientiert sich an der gesunden Seite (Matles-Test) evtl. mit einer minimalen Überkorrektur von $5-10^{\circ}$. Die Nahtstabilität wird durch eine vorsichtige passive Dorsalextension des Fußes getestet. Unbedingt vermieden werden muss eine zu geringe Vorspannung der Sehne und/oder Fehllage der Nähte. Eine Verlängerung der Sehne mit deutlichem Kraftverlust oder eine frühzeitige Reruptur wären die Folgen.

\section{Nachbehandlung}

Einheitliche Standards hinsichtlich der Nachbehandlung gibt es nicht [21]. Die Mehrzahl der Autoren empfiehlt nach operativer Naht zunächst eine Versorgung mit einer Unterschenkelgipsschiene in „entspannter“ Spitzfußstellung für 1-2 Wochen (bis zur gesicherten Wund- heilung), bevor auf einen Orthesenschuh mit Keileinlage (eingestellte Plantarflexion $20-30^{\circ}$ ) für insgesamt 6 Wochen postoperativ gewechselt wird. Abhängig von der Stabilität der Naht darf aus der Orthese die funktionelle Krankengymnastik begonnen werden. Die schrittweise Reduktion der Plantarflexion sowie der Beginn der Vollbelastung wird zeitlich unterschiedlich gehandhabt. In der Regel ist nach 6 Wochen die Vollbelastung erlaubt. Der Beginn mit sportlichen Aktivitäten ist frühestens nach 3 Monaten sinnvoll und individuell festzulegen.

\section{Ergebnisse}

Auch die moderne operative Behandlung der frischen Achillessehnenruptur kann Risiken wie Kraftminderungen, Bewegungseinschränkungen, Rerupturen, Ver- letzungen des N. suralis oder Wundheilungsstörungen nicht vollständig ausschließen.

Nach offener Sehnennnaht ist das Risiko, eine Reruptur zu erleiden, mit 2-4\% gering [17]. Das ursprüngliche Aktivitätsniveau, mit Einschränkung gilt das auch für das Sportniveau, erreichen etwa $80 \%$ der Patienten [12]. Der entscheidende Nachteil der offenen Sehnennaht ist das relativ hohe Infektionsrisiko, das jedoch von früher $20 \%$ auf etwa $11 \%$ gesenkt werden konnte. Besonders gefährdet sind Raucher, Patienten mit Durchblutungsstörungen und Diabetiker $[14,19]$. Dennoch wird in der Literatur über eine hohe Patientenzufriedenheit berichtet und die offene End-zu-End-Naht, kombiniert mit einem Frührehabilitations- 
protokoll, für sportlich aktive Patienten empfohlen [11, 14].

Entscheidender Nachteil der offenen Sehnennaht ist das relativ hohe Infektionsrisiko.

Die Weiterentwicklung der perkutanen Nahttechniken konnten die Ergebnisse deutlich verbessern. So belegen aktuelle Daten eine Verletzungsrate des N. suralis von nur noch $2-3 \%$ (früher $>15 \%$ ), eine Senkung der Reruptur- und Infektrate auf $2-3 \%$. Subjektiv beurteilen die $\mathrm{Pa}-$ tienten ihre Ergebnisse in über $90 \%$ der Fälle mit gut bis sehr gut [11].

Die Ergebnisse der perkutanen Nahttechniken haben sich deutlich verbessert.

Im Vergleich perkutane Nahttechnik mit offener Technik lassen sich in der aktuellen Literatur keine statistisch signifikanten Unterschiede hinsichtlich der postoperativen Komplikationen feststellen. Ding et al. [6] berichten, dass die Patienten mit minimalinvasiver Naht signifikant häufiger sehr gute Ergebnisse zeigen als nach offener Naht (98 vs. 91\%).

\section{Schlussfolgerung}

Sowohl mit operativen als auch mit konservativen Therapieformen sind sehr gute Ergebnisse möglich. Auch die aktuelle Literatur kann keine eindeutige Therapieempfehlung geben. Daher müssen bei der Therapieplanung einer frischen Achillessehnenruptur immer beide Möglichkeiten angesprochen und letztendlich an den individuellen Patientenbedürfnissen ausgerichtet werden.

Folgender Behandlungspfad der frischen Achillessehnenruptur kann anhand der aktuellen Literatur und unserer eigenen klinischen Erfahrungen empfohlen werden: konservativ-funktionelle Therapie:

- Risikopatient: lokal/allgemein erhöhtes OP-Risiko

- bei Rupturen im muskulotendinösen Übergang und bei Teilrupturen

- wenn die Therapie innerhalb $24 \mathrm{~h}$ nach der Ruptur beginnen kann.

- Adaption der Sehnenenden (dynamische Sonografie) bis $20^{\circ}$ Plantarflexion

offene End-zu-End-Naht:

- keine exakte Adaption der Sehnenenden (Spalt > $10 \mathrm{~mm}$ )

- ältere Ruptur (>1 Woche)

- Zusatzmaßnahmen erforderlich (Augmentation, Anfrischung der Sehnenenden)

kombiniert offen-perkutan:

- distale Rupturen (Ankerfixation)

perkutane Naht:

- OP in der 1 . Woche möglich

- Adaption der Sehnenenden (Spalt $>10 \mathrm{~mm}$ )

- Patienten mit Belastungsanspruch (Beruf, Sport)

\section{Literatur}

${ }^{1}$ Amlang MH, Maffuli N, Longo UG et al. Operative Behandlung der Achillessehnenruptur. Unfallchirurg 2010; 113: 712-720

2 Amlang MH, Christiani P, Heinz P et al. Die perkutane Achillessehnennaht mit dem Dresdner Instrument. Technik und Ergebnisse. Unfallchirurg 2005; 108: 529-536

${ }^{3}$ Bauer G, Eberhardt O. Die frische Achillessehnenruptur - Epidemiologie - Ätiologie Diagnostik und aktuelle Therapiemöglichkeiten. Sportverl Sportschad 1999; 13: 79-89

${ }^{4}$ Boorboor P, Lahoda LU, Spies M et al. Resektion der infizierten Achillessehne. Ergebnisse nach plastischer Defektdeckung ohne Achillessehnenersatz. Chirurg 2006; 77: 11441151

${ }^{5}$ Boyden EM, Kitaoka HB, Cahalan TD et al. Late versus early repair of Achilles tendon rupture. Clinical and biomechanical evaluation. Clin Orthop Relat Res 1995; 317: 150-158

${ }^{6}$ Ding WG, LI H, Zhu YP et al. Comparison between tenocutaneous suture and Kessler suture techniques in treating acute closed Achilles tendon rupture. Foot Ankle Surg 2014; 20: 105-108
${ }^{7}$ Del Buono A, Volpin A, Mafffuli N. Minimally invasive versus open surgery for acute Achilles tendon rupture: a systematic review. Br Med Bull 2014; 109: 45-54

8 Gianetti S, Patricola AA, Stancati A et al. Intraoperative ultrasound assistance for percutaneous repair oft he acute Achilles tendon rupture. Orthopedics 2014; 37: 820-824

9 Gulati V,Jaggart M, Al-Nammari SS et al. Management of achilles tendon injury: a current concepts systematic review. World J Orthop 2015; 18: 360-366

${ }^{10}$ Hsu AR, Jones CP, Cohen BE et al. Clinical outcomes and complications of percutaneous achilles repair system versus open technique for acute Achilles tendon ruptures. Foot Ankle Int 2015; 36: 1279-1286

11 Hüfner T, Gaulke R, Imrecke J et al. Konservativ-funktionelle Behandlung der Achillessehnenruptur. Unfallchirurg 2010; 113: 699-704

12 Hüfner T, Krettek C, Knobloch K. Achillessehnenruptur. Fuss Sprungg 2007; 5: 269-277

${ }^{13}$ Huttunen TT, Kannus P, Rolf $C$ et al. Acute achilles tendon ruptures: incidence of injury and surgery in Sweden between 2001 and 2012. Am J Sports Med 2014; 42: 2419-2423

${ }^{14}$ Khan RIK, Smith RLC. Surgical interventions for treating acute Achilles tendon ruptures. Cochrane Database Syst Rev 2010; 9: 1-36

15 Lill H, Moor C, Schmidt A et al. Current status of treatment of Achilles tendon ruptures. Results of a nationwide survey in Germany. Chirurg 1996: 67: 1160-1165

${ }^{16}$ Ma GWC, Griffith TG. Percutaneous repair of acute closed ruptured Achilles tendon. Clin Orthop Relat Res 1977; 128: 247-255

17 Pagenstert G, Leumann A, Frigg A et al. Achillessehnen- und Tibialis-anterior-Sehnenruptur. Orthopäde 2010; 39: 1135-1147

${ }^{18}$ Raikin SM, Garras DN, Krapchev PV. Achilles tendon injuries in a United States population. Foot Ankle Int 2013; 34: 475-480

${ }^{19}$ Strauss EJ, Jshak C, Jazrawi L et al. Operative treatment of acute Achilles tendon ruptures: an institutional review of clinical outcome. 2007; Injury 38: 832-838

${ }^{20}$ Thermann H, Hufner T, Tscherne H. [Achilles tendon rupture]. Orthopäde 2000; 29: $235-$ 250

${ }^{21}$ Ulmar B, Simon S, Eschler A et al. Die Achillessehnenruptur. Unfallchirurg 2014; 117: $921-$ 939

Prof. Dr. med. Gerhard J. Bauer Ärztlicher Direktor a.D. Sportklinik Stuttgart GmbH

c/o SpOrt Medizin Stuttgart GmbH Fritz-Walter-Weg 19

70372 Stuttgart

gj.bauer@icloud.com 